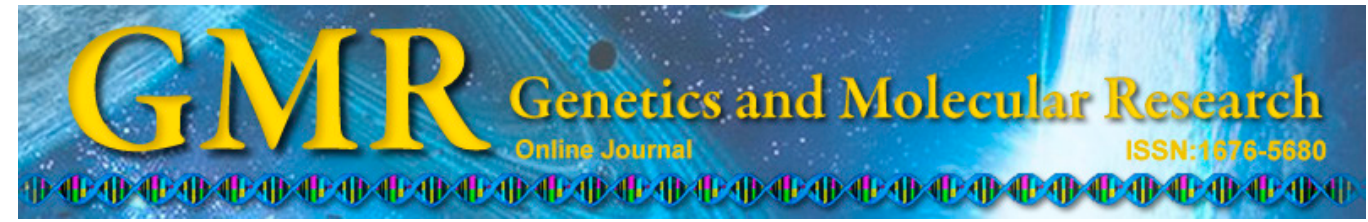

\title{
Heterozygosities and genetic relationship of tea cultivars revealed by simple sequence repeat markers and implications for breeding and genetic mapping programs
}

\author{
L.Q. Tan', C.C. Zhang', G.N. Qi' ${ }^{1}$ L.Y. Wang ${ }^{2,3}$, K. Wei ${ }^{2,3}$, S.X. Chen ${ }^{1}$, \\ Y. Zou ${ }^{1}$, L.Y. Wu ${ }^{2}$ and H. Cheng ${ }^{2,3}$ \\ ${ }^{1}$ Tea Science Department, College of Horticulture, \\ Sichuan Agricultural University, Ya'an, Sichuan, China \\ ${ }^{2}$ National Center for Tea Improvement, Tea Research Institute, \\ Chinese Academy of Agricultural Science, Hangzhou, Zhejiang, China \\ ${ }^{3}$ Key Laboratory of Tea Plant Biology and Resources Utilization, \\ Ministry of Agriculture, Hangzhou, Zhejiang, China \\ Corresponding authors: G.N. Qi / H. Cheng \\ E-mail: guinian5612@163.com / chenghao@mail.tricaas.com
}

Genet. Mol. Res. 14 (1): 1557-1565 (2015)

Received December 19, 2013

Accepted July 3, 2014

Published March 6, 2015

DOI http://dx.doi.org/10.4238/2015.March.6.3

\begin{abstract}
Genetic maps are essential tools for quantitative trait locus analysis and marker-assisted selection breeding. In order to select parents that are highly heterozygous for genetic mapping, the heterozygosity $\left(H_{\mathrm{s}}\right)$ of 24 tea cultivars (Camellia sinensis) was analyzed with 72 simple sequence repeat markers. In total, 359 alleles were obtained with an average of 4.99 per marker. The $H_{\mathrm{S}}$ varied greatly from 37.5 to $71.0 \%$ with an average of $51.3 \%$. On average, tea cultivars from Fujian Province showed a higher level of heterozygosity (59.8\%) than those from Zhejiang (48.5\%) and Yunnan (44.5\%), and the 12 national tea cultivars were generally more heterozygous than the 12 provincial cultivars. Unweighted pair-group analysis using the arithmetic average grouping divided the 24 cultivars into 2 groups that are consistent with the morphological classification. All dual
\end{abstract}


combinations of the 24 cultivars were studied to calculate the percentage of mappable markers when using pseudo-testcross mapping strategy, and results showed that this value also varied greatly from 51.4 to $90.3 \%$. The genetic relationships and $H_{\mathrm{S}}$ differences among different cultivars were discussed, and tea cultivars with high $H_{\mathrm{S}}$ were recommended as cross parents for genetic mapping programs.

Key words: Tea cultivars; Heterozygosity; Genetic mapping; Simple sequence repeat marker

\section{INTRODUCTION}

Tea is a ubiquitous commodity worldwide. In 2012, 3.28 million hectares of tea plant fields were harvested, producing 4.82 million tons of tea (FAO, http://faostat.fao.org/). China has a long tea-growing history; today, 20 of the 34 provinces of China cultivate the tea plant (Yao et al., 2012). In the past 30 years, 124 national tea cultivars (NTCs) have been released to famers in China, and a greater number of provincial tea cultivars (PTCs) have been registered by each tea-growing province (Chen et al., 2007). Investigations of these cultivars with DNA markers may provide useful information for future breeding programs.

Genetic maps are essential tools for implementing quantitative trait locus analysis and marker-assisted selection breeding in the 21st century (Collard and Mackill, 2008). The tea plant [Camellia sinensis (L.) O. Kuntze], is a perennial diploid $(2 \mathrm{n}=30)$ wood plant characterized by a large genome $(\sim 4 \mathrm{~Gb})$ that is self-incompatible and highly heterozygous (Tanaka et al., 2006; Yao et al., 2012). The most used mapping strategy for this kind of plant is the pseudo-testcross strategy that is based on $\mathrm{F}_{1}$ populations, and genetic markers should be heterozygous in at least one of the two cross parents (Grattapaglia and Sederoff, 1994). Theoretically, using a pair of parents with a high degree of heterozygosity could map a large number of markers in a single $\mathrm{F}_{1}$ population and thus reduce the marker-screening cost.

Simple sequence repeat (SSR) or microsatellite markers are very attractive for genetic mapping and other studies because of the following features: codominant, multiple alleles, and high transferability among related species (Schlötterer, 2004). More than 1000 SSR markers have been developed for $C$. sinensis (Freeman et al., 2004; Zhao et al., 2008; Sharma et al., 2009, 2011; Fang et al., 2012; Ma et al., 2010, 2012; Taniguchi et al., 2012a; Yao et al., 2012), and a few SSR-based maps have been reported (Taniguchi et al., 2012b; Tan et al., 2013). In these studies, the average observed heterozygosity $\left(H_{\mathrm{O}}\right)$ of SSR markers varied greatly depending on the marker-developing strategies and plant materials assayed. However, the heterozygosities of different cultivars or germplasms, hereinafter denoted as $H_{\mathrm{s}}$, were seldom discussed. Whether and to what extent the $H_{\mathrm{S}}$ changes in different tea cultivars and among different tea-growing areas and how its value affects the percentage of mappable markers in a certain F1 mapping population are also unknown. We will adress these questions in this paper.

\section{MATERIAL AND METHODS}

\section{Plant materials}

A collection of 24 tea cultivars that mainly originated from 3 tea-growing provinces 
of China was used in this study (Table 1). Among them, 12 were NTCs and the other 12 were PTCs. All of them were landrace or field clone as described by Chen et al. (2007). The young shoots with two leaves and a bud of each cultivar were sampled at the China National Germplasm Hangzhou Tea Repository (Hangzhou, China). The samples were immediately snap frozen in liquid nitrogen and stored at $-70^{\circ} \mathrm{C}$ prior to DNA extraction. Genomic DNA was extracted from the ground tissues using the cetyltrimethylammonium bromide method.

\begin{tabular}{|c|c|c|c|c|c|c|c|}
\hline No. & Name & Origin & Level & No. & Name & Origin & Level \\
\hline 1 & Changyebaihao & Yunnan & PTC & 13 & Pingyangtezao & Zhejiang & PTC \\
\hline 2 & Fudingdabaicha & Fujian & NTC & 14 & Fuandabaicha & Fujian & NTC \\
\hline 3 & Baihaozao & Hunan & NTC & 15 & Meizhan & Fujian & NTC \\
\hline 4 & Longjin43 & Zhejiang & NTC & 16 & Fujianshuixian & Fujian & NTC \\
\hline 5 & Wuniuzao & Zhejiang & PTC & 17 & Rougui & Fujian & PTC \\
\hline 6 & Huangyan & Fujian & NTC & 18 & Foshou & Fujian & PTC \\
\hline 7 & Tieguanyin & Fujian & NTC & 19 & Yunkang14 & Yunnan & NTC \\
\hline 8 & Mingshanbaihao & Sichuan & NTC & 20 & Yunkang43 & Yunnan & PTC \\
\hline 9 & Zhongcha102 & Zhejiang & NTC & 21 & Yunkang27 & Yunnan & PTC \\
\hline 10 & Shuigucha & Zhejiang & PTC & 22 & Yunkang10 & Yunnan & NTC \\
\hline 11 & Huangyezao & Zhejiang, & PTC & 23 & Yunxuan9 & Yunnan & PTC \\
\hline 12 & Anjibaicha & Zhejiang & PTC & 24 & Yungui & Yunnan & PTC \\
\hline
\end{tabular}

NTC: national tea cultivar; PTC: provincial tea cultivar (also see Chen et al., 2007).

\section{SSR markers and amplification}

Seventy-two SSR markers developed by our group previously were used in this study (Table 2; Tan et al., 2013). Polymerase chain reaction (PCR) amplifications were performed in $10-\mu \mathrm{L}$ reaction mixtures containing $1.0 \mu \mathrm{L} 10 \mathrm{X}$ PCR buffer, $0.2 \mu \mathrm{L} 10 \mathrm{mM}$ dNTPs, $0.2 \mu \mathrm{L}$ $10 \mu \mathrm{M}$ of each primer, $0.5 \mathrm{U}$ Taq polymerase (TaKaRa, Dalian, China) and $20 \mathrm{ng}$ template DNA. Thermocycling conditions were as follows: 1) initial denaturation at $94^{\circ} \mathrm{C}$ for $4 \mathrm{~min}$; 2) 35 cycles of $30 \mathrm{~s}$ at $94^{\circ} \mathrm{C}, 30 \mathrm{~s}$ at $58^{\circ} \mathrm{C}$, and $30 \mathrm{~s}$ at $72^{\circ} \mathrm{C}$; and 3) a final extension at $72^{\circ} \mathrm{C}$ for $10 \mathrm{~min}$. PCR products were resolved on $10 \%$ polyacrylamide gels at $150 \mathrm{~V}$ for $90 \mathrm{~min}$ and visualized by silver staining.

\section{Data analysis}

Distinct alleles of the expected size of each marker were scored. The genetic parameters of these markers, including the number of alleles $\left(N_{\mathrm{A}}\right), H_{\mathrm{O}}$, Nei's gene diversity $(H)$, and polymorphism information content (PIC) were estimated by PowerMarker (Liu and Muse, 2005). Nei's genetic identity and genetic distance between two cultivars were calculated by the POPGene program (Yeh and Boyle, 1997). The dendrogram based on unweighted pair-group method using the arithmetic average (UPGMA) was also drawn by POPGene and viewed by MEGA 4.0 (Tamura et al., 2007). The $H_{\mathrm{S}}$ of each cultivar was estimated as $\mathrm{N}_{\mathrm{H}} / \mathrm{N} \mathrm{x} 100 \%$, where $N_{H}$ is the number of heterozygous markers and $N$ is the total assayed marker number. The percentage of mappable markers $\left(P_{\mathrm{M}}\right)$ of each putative parental combination was estimated as $\left(1-\mathrm{N}_{\mathrm{O}} / \mathrm{N}\right) \times 100 \%$, where $N_{O}$ is the number of markers that are homozygous in both parents of that combination. 


\section{RESULTS}

\section{Marker polymorphism}

A total of 359 alleles were detected by the 72 SSR markers (Figure 1). On average, there were 4.99 alleles per locus, and 2 (CsMF1176, CsMF1177, CsMF1256, and CsFM1268) to 12 (CsFM1131) alleles were scored for each marker. The PIC values ranged from 0.042 (CsFM1176) to 0.880 (CsFM1131) with an average of 0.584 . The average $H_{\mathrm{O}}$ and $H$ for all markers tested were 0.513 and 0.628 , respectively. These values indicated that the 72 SSR markers had a high level of polymorphism, and detailed information for each marker is available in Table 2.

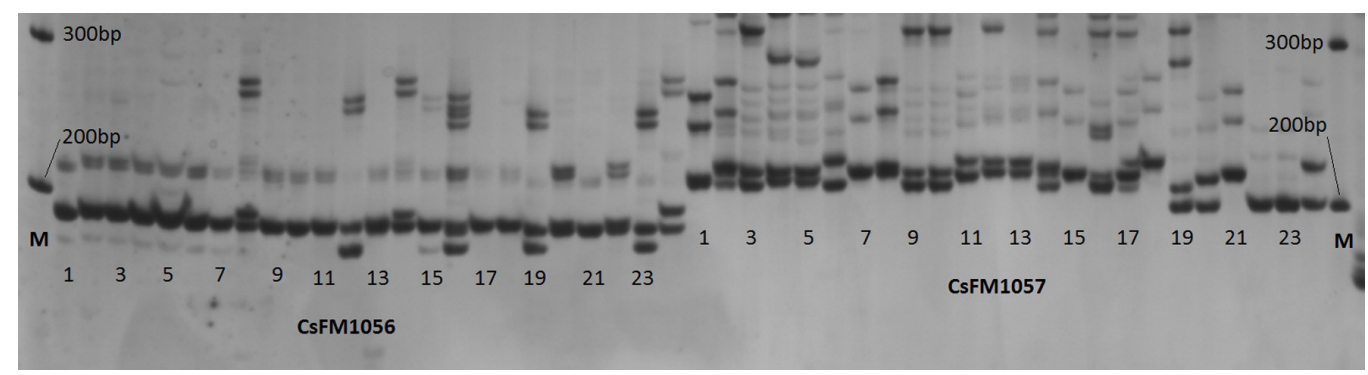

Figure 1. Simple sequence repeat (SSR) allele patterns amplified by CsFM1056 and CsFM1057 in 24 tea cultivars. Polymerase chain reaction (PCR) products were resolved on $10 \%$ polyacrylamide gels and visualized by silver staining. The cultivar names of lanes 1-24 are shown in Table 1. Lane M: DNA ladder.

\section{Genetic relationships and clustering analysis}

The highest Nei's genetic identity (data not shown) was found between cultivars Yunkang27 and Yunxuan9 (0.674), while the lowest identity was found between Changyebaihao and Huangyezao (0.290). The 7 cultivars from Yunnan showed a high level of identity with each other, which ranged from 0.524 to 0.674 . Among the cultivars that are not from Yunnan, the genetic identity between Fudingdabaicha from Fujian and Baihaozao from Hunan was the highest (0.655); this was followed by Meizhan and Rougui (0.612, both from Fujian) and Wuniuzao and Anjibaicha (0.594, both from Zhejiang).

The 24 cultivars were divided into two groups by the UPGMA based on Nei's genetic distances: the 7 cultivars from Yunnan were grouped together and the other 17 were assigned to another group (Figure 2). This result is consistent with the morphological classification. The cultivars from Yunnan belong to $C$. sinensis var. assamica that is characterized by semi-arbor trees and large leaves, while the other 17 cultivars belong to $C$. sinensis var. sinensis that is characterized by shrub trees and small- or moderate-sized leaves (Yao et al., 2012).

\section{$H_{\mathrm{S}}$ of cultivars}

The $H_{\mathrm{S}}$ of each cultivar was defined as the percentage of the heterozygous loci in all loci tested .In this study, the $H_{\mathrm{S}}$ ranged from 37.5 to $71.0 \%$, with an average of $51.3 \%$ (Table 3). The lowest $H_{\mathrm{S}}$ was observed for Changyebaihao (37.5\%) and was followed by Anjibaicha, 
Yunkang27, Yunxuan9, and Yungui (all 40.3\%). The highest $H_{\mathrm{S}}(71.0 \%)$ was scored by Fuandabaicha from Fujian Province. In fact, the average $H_{\mathrm{S}}$ of the 8 cultivars from Fujian Province $(59.8 \%)$ was significantly higher than that from Zhejiang $(48.5 \%, 7$ cultivars) and Yunnan (44.5\%, 7 cultivars).

Table 2. Number of alleles $\left(N_{\mathrm{A}}\right)$, Nei's gene diversity $(H)$, observed heterozygosity $\left(H_{\mathrm{O}}\right)$, and polymorphism information content (PIC) for 72 SSR markers observed in the 24 tea cultivars.

\begin{tabular}{|c|c|c|c|c|c|c|c|c|c|}
\hline Marker & $N_{\mathrm{A}}$ & $H$ & $H_{\mathrm{O}}$ & PIC & Marker & $N_{\mathrm{A}}$ & $H$ & $H_{\mathrm{O}}$ & PIC \\
\hline CsFM1015 & 5 & 0.749 & 0.750 & 0.708 & CsFM1155 & 6 & 0.657 & 0.348 & 0.616 \\
\hline CsFM1026 & 7 & 0.822 & 0.667 & 0.798 & CsFM1156 & 7 & 0.801 & 0.773 & 0.773 \\
\hline CsFM1029 & 7 & 0.712 & 0.583 & 0.682 & CsFM1157 & 4 & 0.554 & 0.542 & 0.471 \\
\hline CsFM1030 & 6 & 0.793 & 0.708 & 0.765 & CsFM1158 & 5 & 0.629 & 0.565 & 0.570 \\
\hline CsFM1031 & 4 & 0.605 & 0.542 & 0.541 & CsFM1159 & 6 & 0.733 & 1.000 & 0.691 \\
\hline CsFM1032 & 8 & 0.765 & 0.542 & 0.730 & CsFM1162 & 5 & 0.622 & 0.500 & 0.581 \\
\hline CsFM1051 & 6 & 0.666 & 0.739 & 0.631 & CsFM1165 & 5 & 0.720 & 0.500 & 0.677 \\
\hline CsFM1056 & 4 & 0.261 & 0.292 & 0.248 & CsFM1166 & 3 & 0.477 & 0.208 & 0.427 \\
\hline CsFM1057 & 6 & 0.788 & 0.625 & 0.759 & CsFM1167 & 7 & 0.826 & 0.792 & 0.804 \\
\hline CsFM1059 & 5 & 0.551 & 0.500 & 0.524 & CsFM1169 & 7 & 0.799 & 0.696 & 0.770 \\
\hline CsFM1061 & 6 & 0.786 & 0.458 & 0.754 & CsFM1170 & 5 & 0.264 & 0.292 & 0.254 \\
\hline CsFM1066 & 4 & 0.424 & 0.522 & 0.392 & CsFM1173 & 6 & 0.687 & 0.375 & 0.652 \\
\hline CsFM1071 & 5 & 0.669 & 0.500 & 0.610 & CsFM1175 & 4 & 0.678 & 0.292 & 0.614 \\
\hline CsFM1078 & 6 & 0.609 & 0.292 & 0.559 & CsFM1176 & 2 & 0.043 & 0.043 & 0.042 \\
\hline CsFM1080 & 3 & 0.398 & 0.333 & 0.354 & CsFM1177 & 2 & 0.325 & 0.136 & 0.272 \\
\hline CsFM1092 & 5 & 0.664 & 0.583 & 0.617 & CsFM1182 & 5 & 0.624 & 0.833 & 0.584 \\
\hline CsFM1100 & 3 & 0.611 & 0.417 & 0.535 & CsFM1186 & 4 & 0.529 & 0.348 & 0.490 \\
\hline CsFM1104 & 6 & 0.669 & 0.625 & 0.626 & CsFM1255 & 4 & 0.730 & 0.458 & 0.680 \\
\hline CsFM1105 & 7 & 0.813 & 0.792 & 0.788 & CsFM1256 & 2 & 0.499 & 0.261 & 0.375 \\
\hline CsFM1107 & 6 & 0.742 & 0.833 & 0.701 & CsFM1261 & 6 & 0.764 & 0.500 & 0.734 \\
\hline CsFM1110 & 8 & 0.811 & 0.542 & 0.784 & CsFM1262 & 3 & 0.559 & 0.542 & 0.488 \\
\hline CsFM1115 & 5 & 0.712 & 0.500 & 0.662 & CsFM1263 & 3 & 0.414 & 0.542 & 0.356 \\
\hline CsFM1116 & 4 & 0.630 & 0.583 & 0.562 & CsFM1267 & 3 & 0.284 & 0.333 & 0.254 \\
\hline CsFM1121 & 7 & 0.774 & 0.500 & 0.744 & CsFM1268 & 2 & 0.194 & 0.217 & 0.175 \\
\hline CsFM1123 & 5 & 0.668 & 0.208 & 0.607 & CsFM1283 & 3 & 0.448 & 0.333 & 0.397 \\
\hline CsFM1125 & 5 & 0.723 & 0.565 & 0.673 & CsFM1286 & 4 & 0.740 & 0.458 & 0.693 \\
\hline CsFM1127 & 4 & 0.705 & 0.250 & 0.649 & CsFM1290 & 6 & 0.658 & 0.833 & 0.617 \\
\hline CsFM1128 & 3 & 0.385 & 0.333 & 0.325 & CsFM1295 & 4 & 0.690 & 0.583 & 0.632 \\
\hline CsFM1129 & 4 & 0.635 & 0.458 & 0.562 & CsFM1307 & 4 & 0.576 & 0.522 & 0.534 \\
\hline CsFM1131 & 12 & 0.891 & 0.792 & 0.880 & CsFM1316 & 4 & 0.676 & 0.667 & 0.617 \\
\hline CsFM1134 & 7 & 0.741 & 0.583 & 0.704 & CsFM1317 & 5 & 0.702 & 0.609 & 0.648 \\
\hline CsFM1135 & 5 & 0.709 & 0.542 & 0.671 & CsFM1322 & 4 & 0.633 & 0.500 & 0.586 \\
\hline CsFM1138 & 6 & 0.718 & 0.458 & 0.679 & CsFM1324 & 4 & 0.666 & 0.552 & 0.613 \\
\hline CsFM1140 & 9 & 0.841 & 0.750 & 0.822 & CsFM1348 & 5 & 0.712 & 0.439 & 0.667 \\
\hline CsFM1143 & 4 & 0.496 & 0.292 & 0.442 & CsFM1351 & 3 & 0.448 & 0.381 & 0.404 \\
\hline CsFM1154 & 5 & 0.687 & 0.625 & 0.629 & CsFM1357 & 5 & 0.631 & 0.650 & 0.589 \\
\hline
\end{tabular}

The 24 cultivars can be classified into 3 groups based on their $H_{\mathrm{S}}$ values: 1) $H_{\mathrm{S}} \geq$ $55 \%$, including 7 NTCs and 2 PTCs; 2) $55 \%>H_{\mathrm{s}} \geq 45 \%$, including 5 NTCs and 3 PTCs; and 3) $H_{\mathrm{S}}<45 \%$, including 7 PTCs. The average $H_{\mathrm{S}}$ of the 12 NTCs $(57.0 \%)$ was notably higher than that of the 12 PTCs $(45.6 \%)$, and the Student $t$-test showed that difference had statistical significance $(\mathrm{P}<0.001)$.

In order to choose the parental combinations that could make the best use of the available SSR marker resources for genetic mapping, we calculated the $P_{\mathrm{M}}$ for all the putative combinations of the 24 cultivars tested. The $P_{\mathrm{M}}$ values for the 276 dual combinations varied from 51.4 (Changyebaihao x Yunkang10) to $90.3 \%$ (Fuandabaicha $\mathrm{x}$ Fudingdabaicha or Rougui), with an average of $72.8 \%$ (Table 3). The average $P_{\mathrm{M}}$ values of each tea cultivar are shown on the diagonal line of Table 3 and Figure 3 and illustrate that they are positively related with the $H_{\mathrm{S}}$ values. 


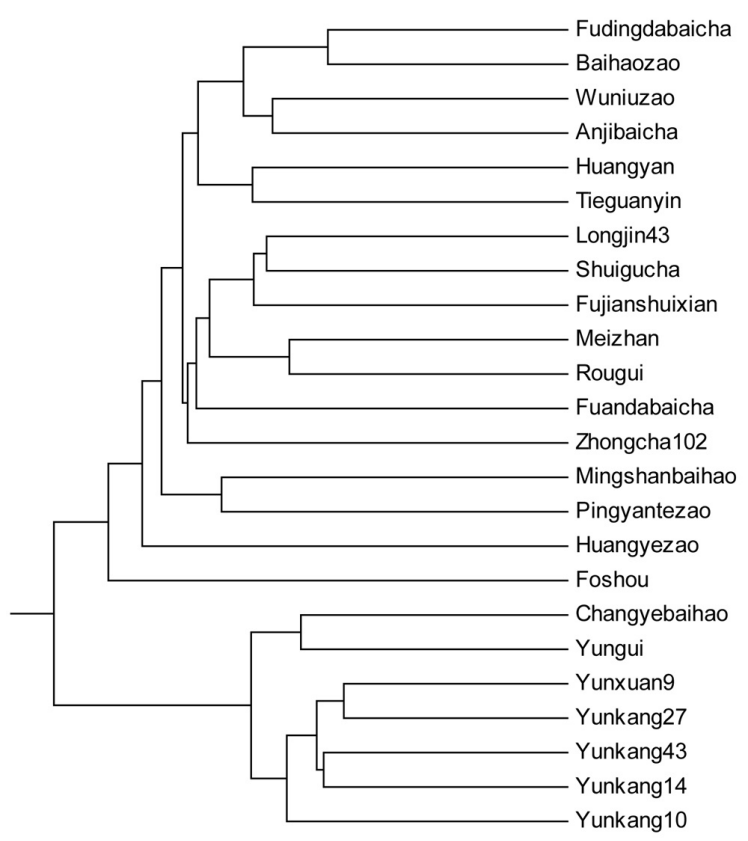

Figure 2. Dendrogram of 24 tea cultivars by the unweighted pair-group method using the arithmetic average (UPGMA) based on information of 72 SSR markers.

Table 3. Heterozygosities $\left(H_{\mathrm{S}}\right)$ of the 24 tea cultivars and percentages of mappable markers $\left(P_{\mathrm{M}}\right)$ of all putative combinations.

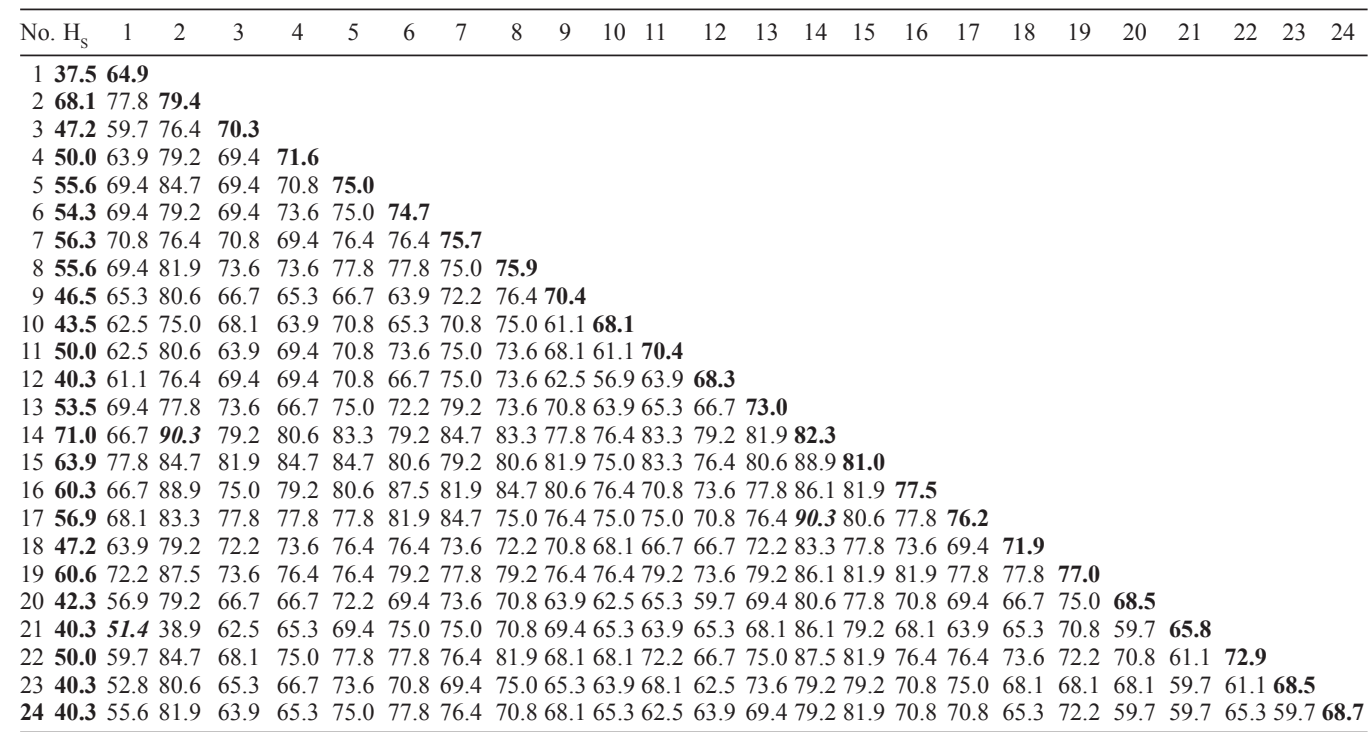

The figures on the diagonal line are the average $P_{\mathrm{M}}$ values of each cultivar, e.g., the figure in the heave gray is the average of the 23 figures in the light gray. 


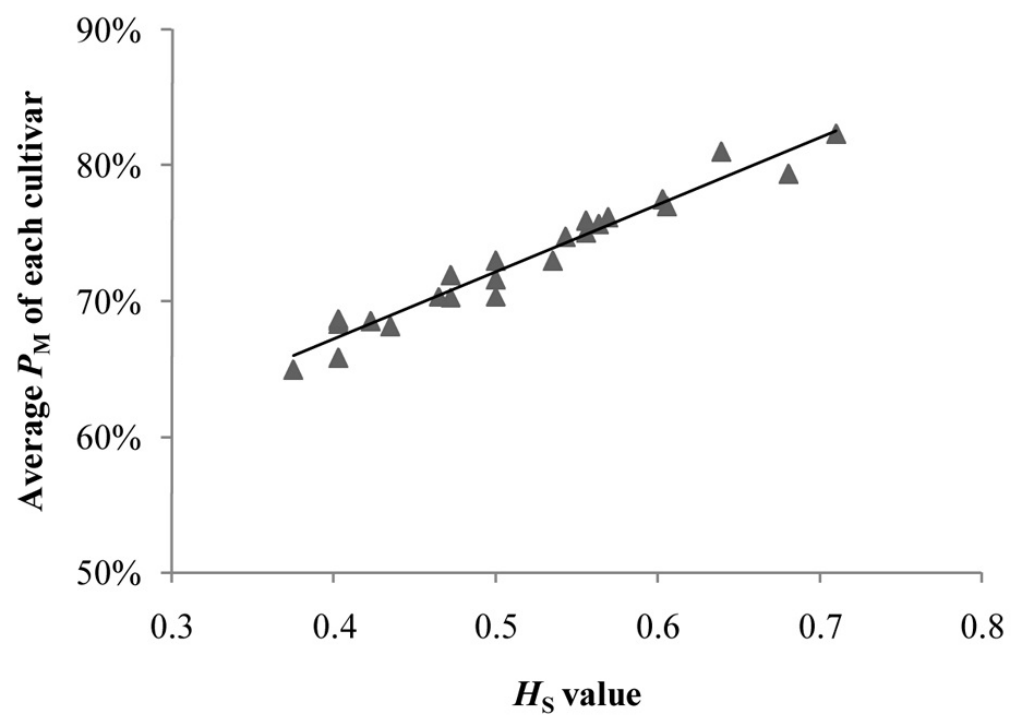

Figure 3. Relationship between the heterozygosity $\left(H_{\mathrm{S}}\right)$ of each cultivar and the average percentage of mappable markers $\left(P_{\mathrm{M}}\right)$.

\section{DISCUSSION}

In this study, we used 72 SSR markers to analyze the genetic relationships and heterozygosity of 24 tea cultivars in China. Overall, a high level of polymorphism was observed for the 72 markers, providing important genetic information as discussed below.

Yunnan is thought to be an original place for $C$. sinensis that has a high level of genetic diversity (Yao et al., 2012). However, the 7 cultivars from this province showed high levels of Nei's genetic identity, indicating close relationships among these cultivars. This is probably because all of these cultivars except Yungui were bred through field clones from the same county (Menghai). Although they originated from different provinces, Fudingdabaicha and Baihaozao also showed a high level of genetic identity (0.655). Another interesting observation is that these two cultivars shared at least one allele at most of the SSR loci tested (data not shown). It is likely that Baihaozao is an offspring of Fudingdabaicha, and this is practically possible because Fudingdabaicha, one of the most outstanding tea cultivars in China, was frequently introduced to other tea-growing areas from Fujian.

Clustering analysis failed to distinguish the cultivars tested geographically in this study except those from Yunnan. This result is consistent with other molecular marker studies of C. sinensis (Chen et al., 2005; Fang et al., 2012; Yao et al., 2012), and this result is probably because genetic exchanges occurred frequently among Fujian, Zhejiang, and Hunan because of human activities.

Genetic heterozygosity is the state of possessing different alleles at a given locus. Generally, $C$. sinensis is thought to be highly heterozygous because of long-term allogamy (Chen et al., 2007). With a set of 72 SSR markers, we showed that the $H_{\mathrm{S}}$ of different tea cultivars varied greatly from 37.5 to $71.0 \%$. A high level of $H_{\mathrm{S}}$ (average $59.8 \%$ ) was observed for the 
cultivars from Fujian Province, suggesting frequent germplasm introductions and hybridizations in this region in history. However, the 7 cultivars from Yunnan showed a relatively low level of $H_{\mathrm{S}}$ (average $44.5 \%$ ). The lowest $H_{\mathrm{S}}$ was scored by Changyebaihao from this province. Furthermore, in an investigation of 178 single nucleotide polymorphism loci, we also observed that Changyebaihao had a lower level of $H_{\mathrm{S}}(37.4 \%)$ compared to the other 6 tea cultivars that ranged from 39.9 to $48.9 \%$ (data not shown). Both the high levels of Nei's genetic identity and the low levels of $H_{\mathrm{S}}$ suggest some degree of inbreeding among the 7 cultivars from Yunnan Province.

Interestingly, the NTCs tended to have a higher $H_{\mathrm{S}}$ than the PTCs. According to the description in the Records of Tea Plant Cultivar in China (The Editorial Board, 2001), NTCs are the primary cultivars that could be spread in a large tea-growing area, while PTCs are supposed to spread mainly within that province. This means that NTCs generally have better fitness than PTCs. Because positive heterozygosity-fitness correlations have been reported for many other plants and animals (Zhang et al., 1996; Hansson and Westerberg, 2002; Chapman et al., 2009), it is possible that there is such a correlation in C. sinensis as well. Further studies with more markers and fitness traits of tea cultivars are needed to investigate this correlationship, which will be of great value in tea-breeding programs if validated.

The long period of juvenility of $C$. sinensis limits the possibility to work on a second generation of hybrids $\left(\mathrm{F}_{2}\right.$ or $\left.\mathrm{BC}\right)$. Consequently, the genetic mapping of $C$. sinensis is normally established in $\mathrm{F}_{1}$ populations using the pseudo-testcross strategy (Grattapaglia and Sederoff, 1994). For the sake of efficient genetic mapping by this strategy, the cross parents of the $F_{1}$ population are required to be highly heterozygous. As mentioned above, there are significant differences in terms of the $H_{\mathrm{S}}$ among the 24 tea cultivars tested. Therefore, it is important to consider the $H_{\mathrm{S}}$ of the cross parents to increase the mapping efficiency. In similar observations of another wood plant, Citrus species, the $P_{\mathrm{M}}$ varied from 21 to $80 \%$ (Luro et al., 2008). In $C$. sinensis, Ma et al. (2012) reported that 25 of 30 SSR markers (83\%) are mappable between a cross of Yingshuan and Baiye Danzhu. Here, we showed that the $P_{\mathrm{M}}$ values for different tea cultivar combinations varied greatly from 51.4 to $90.3 \%$. The cultivars from Fujian Province such as Fuandabaicha, Fudingdabaicha, and Meizhan were recommended as cross parents for genetic mapping, although there are other factors, like fruit-bearing rates and phenotype segregations, that need to be considered when making such choices.

\section{ACKNOWLEDGMENTS}

Research supported by the Twelfth Five-Year Project of Sichuan Province for Tea Plant Breeding, the Science and Technology Department Project of Sichuan Province (\#2012-12CGZHZX0579) and the Earmarked Fund for Modern Agro-Industry Technology Research System (\#nycytx-23).

\section{REFERENCES}

Chapman JR, Nakagawa S, Coltman DW, Slate J, et al. (2009). A quantitative review of heterozygosity-fitness correlations in animal populations. Mol. Ecol. 18: 2746-2765.

Chen L, Gao QK, Chen DM and Xu CJ (2005). The use of RAPD markers for detecting genetic diversity, relationship and molecular identification of Chinese elite tea genetic resources [Camellia sinensis (L.) O. Kuntze] preserved in a tea germplasm repository. Biodivers. Conserv. 14: 1433-1444.

Chen L, Zhou ZX and Yang YJ (2007). Genetic improvement and breeding of tea plant (Camellia sinensis) in China: from individual selection to hybridization and molecular breeding. Euphytica 154: 239-248. 
Collard BCY and Mackill DJ (2008). Marker-assisted selection: an approach for precision plant breeding in the twentyfirst century. Philos. Trans. R. Soc. Lond. B. Biol. Sci. 363: 557-572.

Fang W, Cheng H, Duan Y, Jiang X, et al. (2012). Genetic diversity and relationship of clonal tea (Camellia sinensis) cultivars in China as revealed by SSR markers. Plant Syst. Evol. 298: 469-483.

Freeman S, West J, James C, Lea V, et al. (2004). Isolation and characterization of highly polymorphic microsatellites in tea (Camellia sinensis). Mol. Ecol. Notes 4: 324-326.

Grattapaglia D and Sederoff R (1994). Genetic linkage maps of Eucalyptus grandis and Eucalyptus urophylla using a pseudo-testcross: mapping strategy and RAPD markers. Genetics 137: 1121-1137.

Hansson B and Westerberg L (2002). On the correlation between heterozygosity and fitness in natural populations. Mol. Ecol. 11: 2467-2474.

Liu K and Muse SV (2005). PowerMarker: an integrated analysis environment for genetic marker analysis. Bioinformatics 21: $2128-2129$.

Luro FL, Costantino G, Terol J, Argout X, et al. (2008). Transferability of the EST-SSRs developed on Nules clementine (Citrus clementina Hort ex Tan) to other Citrus species and their effectiveness for genetic mapping. BMC Genomics 9: 287.

Ma JQ, Zhou YH, Ma CL, Yao MZ, et al. (2010). Identification and characterization of 74 novel polymorphic EST-SSR markers in the tea plant, Camellia sinensis (Theaceae). Am. J. Bot. 97: e153-e156.

Ma JQ, Ma CL, Yao MZ, Jin JQ, et al. (2012). Microsatellite markers from tea plant expressed sequence tags (ESTs) and their applicability for cross-species/genera amplification and genetic mapping. Sci. Hortic. 134: 167-175.

Schlötterer C (2004). The evolution of molecular markers - just a matter of fashion? Nat. Rev. Genet. 5: 63-69.

Sharma H, Kumar R, Sharma V, Kumar V, et al. (2011). Identification and cross-species transferability of 112 novel unigene-derived microsatellite markers in tea (Camellia sinensis). Am. J. Bot. 96: e133-e138.

Sharma RK, Bhardwaj P, Negi R, Mohapatra T, et al. (2009). Identification, characterization and utilization of unigene derived microsatellite markers in tea (Camellia sinensis L.). BMC Plant Biol. 9: 53.

Tamura K, Dudley J, Nei M and Kumar S (2007). MEGA4: molecular evolutionary genetics analysis (MEGA) software version 4.0. Mol. Biol. Evol. 24: 1596-1599.

Tan LQ, Wang LY, Wei K, Zhang CC, et al. (2013). Floral transcriptome sequencing for SSR marker development and linkage map construction in the tea plant (Camellia sinensis). PLoS One 8: e81611.

Tanaka J, Taniguchi F, Hirai N and Yamaguchi S (2006). Estimation of the genome size of tea (Camellia sinensis), camellia (C. japonica), and their interspecific hybrids by flow cytometry. Tea Res. J. 101: 1-7.

Taniguchi F, Fukuoka H and Tanaka J (2012a). Expressed sequence tags from organ-specific cDNA libraries of tea (Camellia sinensis) and polymorphisms and transferability of EST-SSRs across Camellia species. Breed. Sci. 62: 186-195.

Taniguchi F, Furukawa K, Ota-Metoku S, Yamaguchi N, et al. (2012b). Construction of a high-density reference linkage map of tea (Camellia sinensis). Breed. Sci. 62: 263-273.

The Editorial Board (2001). Records of China Tea Plant Cultivars. Shanghai Scientific \& Technical Publishers, Shanghai, 15.

Yao MZ, Ma CL, Qiao TT, Jin JQ, et al. (2012). Diversity distribution and population structure of tea germplasms in China revealed by EST-SSR markers. Tree Genet. Genomes 8: 205-220.

Yeh FC and Boyle TJB (1997). Population genetic analysis of co-dominant and dominant markers and quantitative traits. Belg. J. Bot. 129: 157.

Zhang Q, Zhou ZQ, Yang GP, Xu CG, et al. (1996). Molecular marker heterozygosity and hybrid performance in indica and japonica rice. Theor. Appl. Genet. 93: 1218-1224.

Zhao LP, Liu Z, Chen L, Yao MZ, et al. (2008). Generation and characterization of 24 novel EST derived microsatellites from tea plant (Camellia sinensis) and cross-species amplification in its closely related species and varieties. Conserv. Genet. 9: 1327-1331. 